Claremont Colleges

Scholarship@ Claremont

All HMC Faculty Publications and Research

HMC Faculty Scholarship

11-1-1992

\title{
Direct Picosecond Measurement of Photoinduced Cooper Pair Breaking in Lead
}

John F. Federici

ATઐT Bell Laboratories

Benjamin I. Greene

AT $\mathfrak{W}$ T Bell Laboratories

Peter N. Saeta

Harvey Mudd College

Douglas R. Dykaar

AT\&T Bell Laboratories

F. Sharifi

University of California - San Diego

See next page for additional authors

\section{Recommended Citation}

"Direct picosecond measurement of photo-induced Cooper pair breaking in lead," J. F. Federici, B. I. Greene, P. N. Saeta, D. R. Dykaar, F. Sharif, and R. C. Dynes, Phys. Rev. B 46, 11153 (1992).

This Article is brought to you for free and open access by the HMC Faculty Scholarship at Scholarship @ Claremont. It has been accepted for inclusion in All HMC Faculty Publications and Research by an authorized administrator of Scholarship @ Claremont. For more information, please contact scholarship@cuc.claremont.edu. 
Authors

John F. Federici, Benjamin I. Greene, Peter N. Saeta, Douglas R. Dykaar, F. Sharifi, and R. C. Dynes 


\title{
Direct picosecond measurement of photoinduced Cooper-pair breaking in lead
}

\author{
J. F. Federici, B. I. Greene, P. N. Saeta, and D. R. Dykaar \\ AT\&T Bell Laboratories, 600 Mountain Avenue, Murray Hill, New Jersey 07974 \\ F. Sharifi and R. C. Dynes \\ Department of Physics, University of California at San Diego, San Diego, California 92093
}

(Received 4 May 1992)

\begin{abstract}
We report on a direct kinetic measurement of Cooper-pair breaking in superconducting lead. A 100-fs pulse of visible light was used to excite a thin-film lead sample, while the Cooper-pair density was optically probed using an ultrashort pulse of broadband far-infrared radiation. Subsequent to the absorption of the visible light, a rapid $(<1$ ps) change in the far-infrared optical transmission was observed, corresponding to the breaking of Cooper pairs and the collapse of the superconducting gap.
\end{abstract}

Thin superconducting lead films have been extensively studied and characterized using far-infrared (FIR) linear spectroscopy. ${ }^{1-3}$ The experiments described in Ref. 3 were the first to demonstrate a FIR manifestation of a superconducting energy gap. Refinements in the experimental technique improved the signal to noise and led to the observation of experimental anomalies in $\mathrm{Pb}$ films that could be explained by strong-coupled superconducting theory. ${ }^{4}$ Time-resolved studies of ultrashort electrical pulse propagation along striplines have also been used to study the linear spectroscopy of superconductors. ${ }^{5-7}$ These latter measurements have demonstrated dispersion and attenuation of electrical pulses consistent with the presence of quasiparticles and a superconducting energy gap. In addition, linear FIR spectroscopy of high- $T_{c}$ materials has been extensively studied by a variety of techniques over the past few years. ${ }^{8,9}$

Recently, there has been heightened interest in the transient nonlinear spectroscopy of superconductors. Short pulse, visible pump-and-probe optical measurements performed on conventional superconductors at room temperature have inferred electron-phonon coupling constants in excellent agreement with those obtained from tunneling data. ${ }^{10,11}$ In these pump-probe measurements, a 60 -fs pulse of visible light was assumed to heat the electrons instantly to an elevated temperature. Subsequent equilibration of electrons and phonons was monitored by measuring the transient reflectivity with a second visible light pulse. Hot electrons were observed to equilibrate with the phonons in about $1 \mathrm{ps}$ in $\mathrm{Pb}$. Other workers have reported similar measurements on high $T_{c}$ materials. ${ }^{12-14}$

In this paper, we report novel measurements using a visible excitation pulse and a FIR probe pulse performed on thin superconducting $\mathrm{Pb}$ films. Subsequent to the $\mathrm{ab}-$ sorption of visible light, processes involving energy transfer from electrons or phonons lead to the breaking of Cooper pairs. By optically monitoring the spectroscopic region corresponding to the superconducting energy gap $2 \Delta$ with the FIR probe pulse, the excitation dynamics and spectral response of Cooper pairs subsequent to visible excitation were studied. This all-optical technique of studying quasiparticle dynamics has the advan- tage that physical contact to and patterning of the sample (as is necessary with electrical measurements) is not required. Furthermore, the gap region is the most sensitive optical probe of superconducting behavior.

Lead samples were grown as $\mathrm{Ge}-\mathrm{Pb}-\mathrm{Ge}$ sandwiches at $77 \mathrm{~K}$ on crystalline quartz substrates. The $\mathrm{Ge}$ layers were $20 \AA$, while the $\mathrm{Pb}$ layer was typically $50 \AA$. The undercoating of $\mathrm{Ge}$ facilitated the growth of high quality, uniform thin $\mathrm{Pb}$ films, while the Ge cap layer prevented $\mathrm{Pb}$ oxidation. The room-temperature resistance of the films was typically $50 \Omega / \square$, and decreased by a factor of $\sim 3$ on cooling to $10 \mathrm{~K}\left(T_{c} \approx 7 \mathrm{~K}\right)$. Resistivity measurements indicated a sharp superconducting transition. The samples were cooled in a He-exchange gas dewar. The sample chamber could be evacuated to reduce the sample temperature to about $2 \mathrm{~K}$.

The experimental setup for FIR pump-probe spectroscopy has been described in detail elsewhere. ${ }^{15}$ A $10-\mathrm{Hz}$ $\mathrm{Nd}$ : yttrium aluminum garnet-pumped, amplified colliding pulse mode-locked dye laser system was used to generate $500-\mu \mathrm{J}, 100-\mathrm{fs}$ light pulses at $630 \mathrm{~nm}$. The visible light was divided into two beams. Half the light illuminated a semi-insulating GaAs wafer near Brewster's angle. The wafer was externally biased, producing a large $\left(\sim 7 \times 10^{3} \mathrm{~V} / \mathrm{cm}\right)$ electric field in the plane of incidence, to increase the intensity of the generated FIR pulse. The resulting FIR probe pulse consisted of a half cycle of light with a duration of approximately 0.4 ps. The TMpolarized probe pulse was focused with an off-axis parabolic reflector to a spot diameter of roughly $1 \mathrm{~cm}$ and incident at $45^{\circ}$ on the back surface of the substrate. A second parabolic reflector collected the transmitted infared light, which was detected by a bolometer held at 4.2 K. ${ }^{16}$ FIR transmission spectra were obtained by taking the Fourier transform of the first-order autocorrelation of the probe light. The remainder of the visible light was strongly attenuated and used to excite the front face of the $\mathrm{Pb}$ sample at a $45^{\circ}$ angle of incidence. The incident excitation energy density was typically $\sim 1 \mu \mathrm{J} / \mathrm{cm}^{2}$.

Figure 1 shows the ratio $T_{S} / T_{N}$ of the FIR transmission in the superconducting $(2.7 \mathrm{~K})$ and normal $(10 \mathrm{~K})$ state of $\mathrm{Pb}$ in the absence of photoexcitation. As was first observed by Glover and Tinkham, ${ }^{3}$ the transmission ratio 


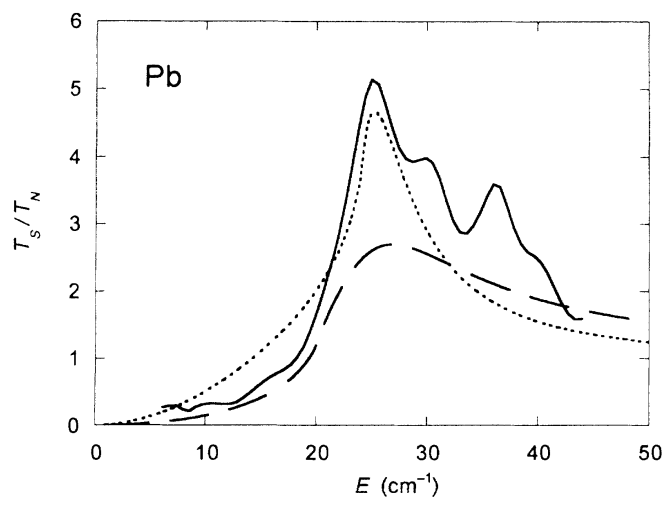

FIG. 1. Ratio of the far-infrared light transmitted through a thin $\mathrm{Pb}$ film in the superconducting state at $2.7 \mathrm{~K}$ as compared to the normal state at $10 \mathrm{~K}$ (solid curve). The oscillations arise from an etalon effect in the detector. The dashed curve was calculated from Eq. (1) and the Mattis-Bardeen expression for $\sigma^{S} / \sigma^{N}$ at zero temperature. The dotted curve was calculated from Eq. (1) using the Leplae expression for $\sigma^{S} / \sigma^{N}$ assuming a Drude conductivity in the normal state.

is peaked near the superconducting energy gap $v_{g}=2 \Delta \approx 22.5 \mathrm{~cm}^{-1}$. Below this frequency, the transmission in the superconducting state is strongly reduced by the large imaginary component of the conductivity, $\sigma_{2}^{s}$, which diverges as $1 / v$ at low frequencies. Because of an unavoidable reflection in the bolometer, the data display an oscillatory artifact that is imperfectly removed in forming the ratio of two spectra, as in Fig. 1. The oscillations are unrelated to phonon structure in the conductivity.

Pump-probe measurements were performed with the sample at $2.7 \mathrm{~K}$ in $\mathrm{He}$ vapor. Figure 2(a) shows the farinfrared transmission of the $\mathrm{Pb}$ film, normalized to the normal state transmission at $10 \mathrm{~K}$, for two values of probe delay with respect to the optical excitation pulse. The fluence of the excitation beam was $\sim 1 \mu \mathrm{J} / \mathrm{cm}^{2}$. For comparison, the data of Fig. 1 with the excitation beam blocked are also shown. On the time scale of a picosecond the transmission peak collapses and the transmission below the energy gap begins to rise. The kinetics of this transformation have been investigated by measuring the total (spectrally integrated) FIR transmission of the film as a function of pump-probe delay. The resulting time trace is shown in Fig. 2(b). The origin of time has been assigned to the midway point of the transmission drop and corresponds to the spectrum labeled 0 ps in Fig. 2(a). The next spectrum, marked +1.3 ps, was taken just after the transmission reached its steady-state value, as indicated in Fig. 2(b). Spectra taken at a delay of $0.7 \mathrm{~ns}$ showed the transmission beginning to recover to the unexcited (superconducting) curve. For excitation fluences of $1 \mu \mathrm{J} / \mathrm{cm}^{2}$ and below, the kinetic response of the sample showed no evidence of saturation.

The instrumental response of our pump-probe apparatus was investigated by replacing the lead film with a polished wafer of semi-insulating silicon. Absorption of the visible excitation light creates free electrons and holes resulting in nearly frequency-independent Drude absorp- tion of the FIR probe light. ${ }^{15,17}$ The delay of the excitation light was scanned and the change in FIR transmission of the $\mathrm{Si}$ recorded. The resulting curve was essentially identical to Fig. 2(b). The temporal resolution was also estimated directly from the measured FIR autocorrelation. Assuming that the FIR pulse is symmetric in time, its temporal profile $I(t)$ can be computed directly. ${ }^{16}$ The rise time of the integral of this profile was found to be about half the measured value for $\mathrm{Si}$ and $\mathrm{Pb}$. The most probable cause of this difference is alignment. Noncollinearity of the excitation pulse with respect to the FIR probe of $1^{\circ}$ leads to a spread of 0.6 ps across the 1 $\mathrm{cm}$ probe beam. The measured kinetic response time, therefore, represents an upper bound on the actual response time.

The transmission of a thin metallic film of conductivity $\sigma$ on a transparent substrate of index $n$ is given by the expression $^{18}$

$$
T=\left|1+\frac{\sigma d Z_{0}}{n+1}\right|^{-2},
$$

where $d$ is the film thickness, $Z_{0}$ is the impedance of free space ( $377 \Omega, \mathrm{mks} ; 4 \pi / c, \mathrm{cgs})$, and it is assumed that mul-
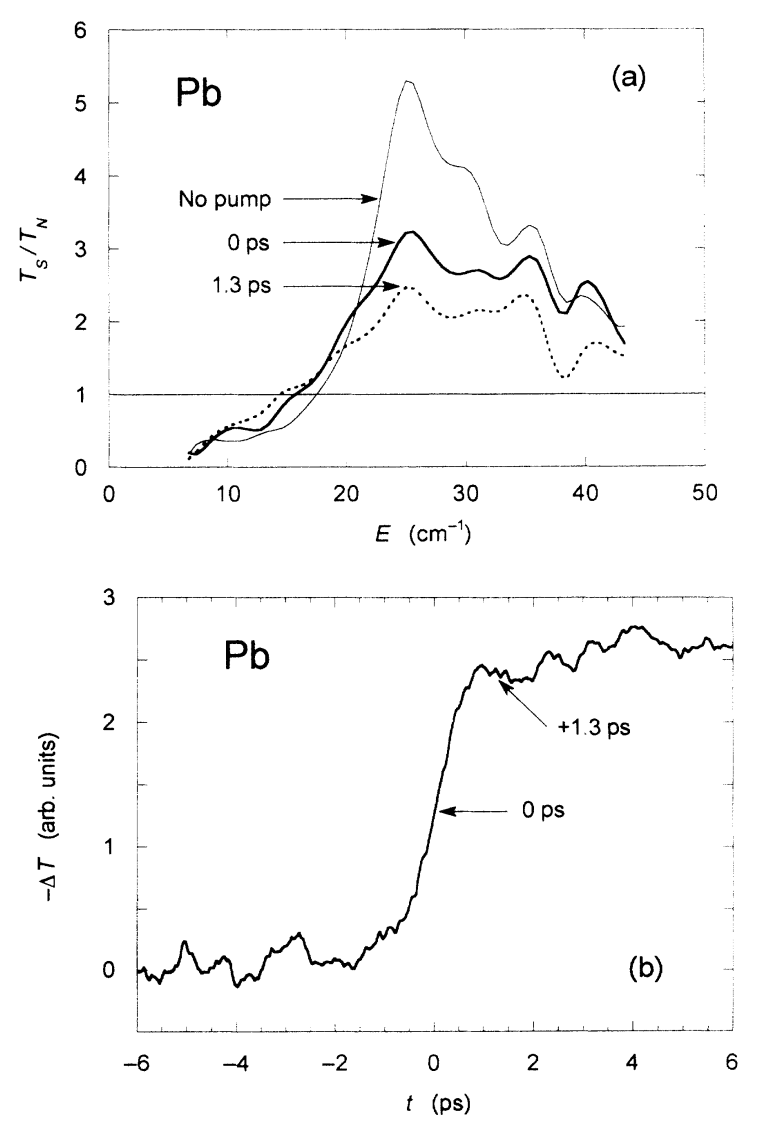

FIG. 2. (a) Spectrum of transmitted infrared light at various delays of the infrared probing pulse with respect to the 2-eV excitation pulse. The film is held at $2.7 \mathrm{~K}$ and excited with an incident fluence of $\sim 1 \mu \mathrm{J} / \mathrm{cm}^{2}$. (b) Change in the spectrally integrated infrared transmission as a function of delay between visible excitation and infrared probe pulses. 
tiple reflections in the substrate can be neglected. In the limit of vanishing film thickness, the normal-state conductivity is dominated by surface scattering and is approximately real and independent of frequency. ${ }^{3}$ Mattis and Bardeen have derived an expression for the conductivity of a BCS superconductor in this "extreme dirty limit," 19 which was found to agree quite well with transmission measurements on $\mathrm{Pb}$ films having dc resistances of $\approx 200 \Omega / \square .^{3}$ For our $\mathrm{Pb}$ films with $R \approx 20 \Omega / \square$ at 10 $K$, the Mattis-Bardeen expression underestimates the peak height, as shown in Fig. 1. This indicates that our films are thick enough and of sufficient quality that the normal-state conductivity is not totally dominated by surface scattering. Leplae has derived a general expression for the conductivity of a film in the superconducting state in terms of the frequency-dependent normal-state conductivity. $^{20}$ Using a simple Drude form for the normal-state conductivity, $\sigma^{N}(\omega)=\sigma_{0} /(1-i \omega \tau)$, where $\sigma_{0}$ is the de conductivity and $\tau$ is the Drude scattering time, McKnight, Bean, and Perowitz found that the Leplae expression gave a transmission peak that was both higher and narrower than the Mattis-Bardeen expression, in good agreement with their transmission data on thick $\mathrm{V}_{3}$ Si films. ${ }^{21}$ Using the same Drude expression for $\sigma^{N}$, we have attempted to fit our data to the Leplae expression with $\tau=0.35 \mathrm{ps}$, as shown by the dotted line in Fig. 1 . Despite the poor quality of the fit, it is clear that a frequency-dependent $\sigma^{N}$ leads to a narrower transmission peak of greater height, in general agreement with our data. Furthermore, the large peak above the gap and the prominent loss of transmission in the gap unambiguously identify the presence of Cooper pairs in the superconducting phase.

Absorption of 2-eV photons from the excitation pulse deposits energy in the electronic system within the $100-\mathrm{fs}$ duration of the pulse. The time scale for electronelectron interactions is much shorter than this, given roughly by the inverse of the plasma frequency. Thus it is commonly assumed ${ }^{10,11}$ that immediately following the excitation pulse the electrons can be described by a thermal distribution at an elevated temperature $T_{e}$, while the lattice temperature $T_{L}$ remains unchanged. $T_{e}$ can be estimated from the absorbed energy density $u \approx 0.2 \mathrm{~J} \mathrm{~cm}^{-3}$ and the electronic specific heat $c=\gamma T_{e}$, where $\gamma=3.0 \mathrm{~mJ} \mathrm{~mol}^{-1} \mathrm{~K}^{-2,22}$ giving $30-50 \mathrm{~K}$ for an excitation fluence of $1 \mu \mathrm{J} / \mathrm{cm}^{2}$. Recent time-of-flight measurements of photoemitted electrons in $\mathrm{Au}$ show, however, that thermalization of the electron distribution in a good metal can take longer than $1 \mathrm{ps.}^{23}$ It therefore may be inaccurate to characterize the electron distribution as thermal on the subpicosecond time scale and $T_{e}$ should be understood as the absorbed energy density.

Electron-phonon coupling transfers energy from electrons to phonons with a characteristic time of $\sim 0.5 \mathrm{ps}$ in $\mathrm{Pb},{ }^{11}$ producing an equilibrium temperature in the range 6-9 $\mathrm{K}$ within a few picoseconds. It should be noted that the energy of the FIR probe is more than an order of magnitude lower than the excitation pulse energy and causes negligible heating of the sample. The ability to observe a superconducting spectrum from the unexcited sample (Fig. 1) provides simple proof that the probe pulse contains insufficient energy to significantly perturb the sample. Thus, an equilibrium analysis suggests that the electron temperature immediately following excitation is many times $T_{c}$ and that within a few picoseconds the equilibrated sample temperature is near $T_{c}$.

In principle, the loss of superconducting behavior involves two processes: the loss of coherence among the paired electrons near the Fermi energy and the closing of the energy gap. At long times, so that complete equilibration between electrons and phonons takes place, it is clear that if the film is heated above $T_{c}$ it will exhibit the conductivity and FIR transmission of the normal state. At short times, however, it is conceivable that the initial 2.7-K value of the energy gap remains frozen in and that some vestiges of the macroscopic coherent state persists. Our experimental results [Fig. 2(b)] have direct bearing on this question and suggest an upper bound of $\sim 1$ ps on the closing of the gap in the electric density of states.

In conclusion, we have directly measured the process of Cooper-pair breaking in a superconductor via a novel combination of visible excitation and a short-pulse broadband FIR probe. This direct measure of the temporal response of the electronic state of the superconductor allows us to study the dynamics of the quasiparticle and Cooper pairs on the picosecond time scale. Our timeresolved FIR transmission data indicate that subsequent to optical excitation the superconducting gap closes in less than one picosecond.

The authors gratefully thank G. A. Thomas, D. H. Rapkine, S. Schmitt-Rink, P. B. Littlewood, and A. J. Millis for many valuable discussions.
${ }^{1} \mathrm{~S}$. Perkowitz, in Infrared and Millimeter Waves, edited by K. J. Button (Academic, New York, 1983).

${ }^{2}$ L. H. Palmer and M. Tinkham, Phys. Rev. 165, 588 (1968).

${ }^{3}$ R. E. Glover, III and M. Tinkham, Phys. Rev. 108, 243 (1957).

${ }^{4}$ R. R. Joyce and P. L. Richards, Phys. Rev. Lett. 24, 1007 (1970).

${ }^{5}$ W. J. Gallahger, C.-C. Chi, I. N. Duling, III, D. Grischkowsky, N. J. Halas, M. B. Ketchen, and A. W. Kleinsasser, Appl. Phys. Lett. 50, 350 (1987).

${ }^{6}$ M. C. Nuss and K. W. Goossen, IEEE J. Quantum Electron.
25, 2596 (1989).

7J. F. Whitaker, R. Sobolewski, D. R. Dykaar, T. Y. Hsiang, and G. A. Mourou, IEEE Trans. Microwave Theory Tech. 36, 277 (1988).

${ }^{8}$ T. Timusk and D. B. Tanner, Physical Properties of HighTemperature Superconductors, I (World Scientific, Singapore, 1989).

9J. Orenstein, G. A. Thomas, A. J. Millis, S. L. Cooper, D. H. Rapkine, and T. Timusk, Phys. Rev. B 42, 6342 (1990).

${ }^{10}$ P. B. Allen, Phys. Rev. Lett. 59, 1460 (1987). 
${ }^{11}$ S. D. Brorson, A. Kazeroonian, J. S. Moodera, D. W. Face, T. K. Cheng, E. P. Ippen, M. S. Dresselhaus, and G. Dresselhaus, Phys. Rev. Lett. 64, 2172 (1990).

${ }^{12}$ S. G. Han, K. S. Wong, Z. V. Vardeny, O. G. Symko, and G. Koren, Bull. Am. Phys. Soc. 35, 678 (1990).

${ }^{13}$ G. L. Eesley, J. Heremans, M. S. Meyer, G. L. Doll, and S. H. Liou, Phys. Rev. Lett. 65, 3446 (1990).

${ }^{14}$ J. Chwalek, C. Uher, J. F. Whitaker, G. A. Mourou, J. Agostinelli, and M. Lelenthal, Appl. Phys. Lett. 57, 1696 (1990).

${ }^{15}$ P. N. Saeta, J. F. Federici, B. I. Greene, and D. R. Dykaar, Appl. Phys. Lett. 60, 1477 (1992).

${ }^{16}$ B. I. Greene, J. F. Federici, D. R. Dykaar, R. R. Jones, and P.
H. Bucksbaum, Appl. Phys. Lett. 59, 893 (1991).

${ }^{17}$ B. I. Greene, J. F. Federici, D. R. Dykaar, A. F. J. Levi, and L. Pfeiffer, Opt. Lett. 16, 48 (1991).

${ }^{18}$ M. Tinkham, Phys. Rev. 104, 845 (1956).

${ }^{19}$ D. C. Mattis and J. Bardeen, Phys. Rev. 111, 412 (1958).

${ }^{20}$ L. Leplae, Phys. Rev. B 27, 1911 (1983).

${ }^{21}$ S. W. McKnight, B. L. Bean, and S. Perkowitz, Phys. Rev. B 19, 1437 (1979).

${ }^{22}$ B. J. C. van der Hoeven, Jr. and P. H. Keesom, Phys. Rev. 137, A103 (1965).

${ }^{23}$ W. S. Fann, Bull. Am. Phys. Soc. 37, 158 (1992). 